\title{
Genome-wide identification and expression profile analysis of the Hsp20 gene family in Barley (Hordeum vulgare L.)
}

Jie Li ${ }^{1}$, Xinhao Liu ${ }^{\text {Corresp. } 2}$

${ }^{1}$ College of Agronomy, Xinyang Agriculture and Forestry University, Xinyang, Henan Province, China

2 Kaifeng Agriculture and Forestry Science Institute, Kaifeng, Henan Province, China

Corresponding Author: Xinhao Liu

Email address: IxhIxhlj12@126.com

In plants, heat shock proteins (Hsps) play important roles in response to diverse stresses. Hsp20 is the major family of heat shock proteins, but their role remains poorly understood in barley (Hordeum vulgare L.). To reveal the mechanisms of barley Hsp20s response to stress conditions, we performed a comprehensive genome-wide analysis of the barley Hsp20 gene family using bioinformatics-based methods. In total, 38 putative barley Hsp20 genes (HvHsp20s) were identified in barley and grouped into four subfamilies (C, CP, PX, and MT) based on predicted subcellular localization and their phylogenetic relationships. A sequence analysis indicated that most Hv Hsp20 genes have no intron or one with a relatively short length. In addition, the same group of HvHsp20 proteins in the phylogenetic tree shared similar gene structure and motifs, indicating that they were highly conserved and might have similar function. Based on RNA-seq data analysis, we showed that the transcript levels of HvHsp20 genes could be induced largely by abiotic and biotic stresses such as heat, salt, and powdery mildew. Three HvHsp20 genes, HORVU7Hr1G036540, HORVU7Hr1G036470, and HORVU3Hr1G007500, were up-regulated under biotic and abiotic stresses, suggesting their potential roles in mediating the response of barley plants to environment stresses. These results provide valuable information for further understanding the complex mechanisms of $\mathrm{HvHsp2O}$ gene family in barley. 
1 Genome-wide identification and expression profile analysis of the Hsp20

2

3

4

5

6

7

8

9

10

$11{ }^{*}$ Correspondence: lxhlxhlj12@126.com.

12

13

14

15

16

17

18

19

20

21

22

23

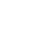

\section{Author affiliation:} China

10

2

3

4

15

16

19

23 gene family in Barley (Hordeum vulgare L.)

Jie $\mathrm{Li}^{1}$, Xinhao $\mathrm{Liu}^{2 *}$

${ }^{1}$ College of Agronomy, Xinyang Agriculture and Forestry University, Xinyang, Henan 464001,

${ }^{2}$ Kaifeng Agriculture and Forestry Science Institute, Kaifeng, Henan 475004, China 


\section{ABSTRACT}

In plants, heat shock proteins (Hsps) play important roles in response to diverse stresses. Hsp20 is the major family of heat shock proteins, but their role remains poorly understood in barley (Hordeum vulgare L.). To reveal the mechanisms of barley Hsp20s response to stress conditions, we performed a comprehensive genome-wide analysis of the barley Hsp20 gene family using bioinformatics-based methods. In total, 38 putative barley Hsp20 genes (HvHsp20s) were identified in barley and grouped into four subfamilies $(\mathrm{C}, \mathrm{CP}, \mathrm{PX}$, and MT) based on predicted subcellular localization and their phylogenetic relationships. A sequence analysis indicated that most HvHsp20 genes have no intron or one with a relatively short length. In addition, the same group of HvHsp20 proteins in the phylogenetic tree shared similar gene structure and motifs, indicating that they were highly conserved and might have similar function. Based on RNA-seq data analysis, we showed that the transcript levels of $H v H s p 20$ genes could be induced largely by abiotic and biotic stresses such as heat, salt, and powdery mildew. Three HvHsp20 genes, HORVU7Hr1G036540, HORVU7Hr1G036470, and HORVU3Hr1G007500, were up-regulated under biotic and abiotic stresses, suggesting their potential roles in mediating the response of barley plants to environment stresses. These results provide valuable information for further understanding the complex mechanisms of $H v H s p 20$ gene family in barley. 
INTRODUCTION

Plants are exposed to a variety of biotic and abiotic stresses which cause disruption of protein homeostasis (Yuko and Shunji 2013). Maintenance in functional native conformations of proteins is important for cell survival under stress. Heat shock proteins (Hsps) exist widely in prokaryotic and eukaryotic cells, are induced by many stresses (such as hot temperatures, drought, cold and various pathogen attacks, etc.), are essential components contributing to cellular homeostasis under stress conditions (Shoseyov 2004; Park and Seo 2015).

57 Many Hsps are located in the cytoplasm and nucleus, and others are located in plastids, mitochondria, chloroplasts, endoplasmic reticulum or peroxisomes, suggesting they play different roles in protein homeostasis (Scharf et al. 2001). Based on protein molecular weight and sequence homology, the plant Hsps are classified into five major families: Hsp100, Hsp90, Hsp70, Hsp60 and small Hsps (sHsps) with molecular sizes ranging from 15 to $42 \mathrm{kDa}$ (Shoseyov 2004). Because most of sHsps molecular weights range from 15-22 kDa, which are also called Hsp20 family.

Hsp20s function as molecular chaperones by binding to partially folded or denatured proteins to prevent proteins from irreversible aggregation and keep them stable (Eyles and Gierasch 2010, Sun et al. 2002). Hsp20s possess a conserved sequence of 80-100 amino acid residues called $\alpha$-crystallin domain (ACD) in the C-terminal region (Scharf et al. 2001). The highly conserved ACD is flanked by a variable N-terminal domain and a short C-terminal extension. The three different regions have different functions. The N-terminal region 
70 participates in substrate binding, the ACD is involved in substrate interactions, while the C-

71 terminus extension is responsible for homo-oligomerization (Giese and Vierling 2004; Jaya et al.

72 2009). This ACD domain is comprised by two compact hydrophobic $\beta$-strand structures that are

73 separated by $\alpha$-helical region of variable length (Bondino et al. 2012).However, not all Hsp20s

that contain an ACD domain, and so named ACD proteins, belonged to Hsp20s because certain

ACD proteins are known to have different functions (Bondino et al. 2012). In plants, Hsp20s are

encoded by nuclear genes. Based on the subcellular localization, sequence homology and

function, Hsp20s are divided into various subfamilies (CI-CVI, MTI, MTII, ER, CP and PX). CI-

CVI subfamilies localize to the cytoplasm/nucleus, MTI and MTII subfamilies localize to mitochondria, ER, CP, and PX localize to the endoplasmic reticulum, chloroplast, and peroxisome, respectively (Waters 2013). To date, the $H s p 20$ gene families have been reported in many plants, such as 13 Hsp20 genes were identified in Arabidopsis (Scharf et al. 2001a), 23 Hsp20 genes in rice (Sarkar et al. 2009), and 163 Hsp20 candidates in wheat (Muthusamy et al. 2017). Furthermore, the biologic function of Hsp20s in protecting plants under various stress conditions is well documented in several plants including soybean (Lopescaitar et al. 2013), tomato (Yu et al. 2016a), and pepper (Guo et al. 2015). and identified 20 sHsp genes (Reddy et al. 2014). Last year, a high-quality reference genome assembly for barley was presented, which provides a convenience for further understanding the barley Hsp20 gene family (Mascher et al. 2017). In the present study, $38 H v H s p 20$ genes were identified using the new barley genome by bioinformatics methods. Then the analysis of identified HvHsp20 genes in the sequence features, chromosomal locations, phylogenetic relationships, and dynamic expression patterns in response to biotic and abiotic stress conditions 
93 were conducted, which provided valuable information for further investigations of the barley

94 Hsp20 gene family.

95

MATERIALS AND METHODS

Genome-wide identification of Hsp20 proteins and chromosomal location

The barley genome sequences were downloaded from Ensembl database (http://plants.ensembl.org). The Hsp20 candidates were identified as the methods of Lozano et al. (2015). The HMMER software (http://hmmer.janelia.org) was used to build the predicted barley protein data. The Hidden Markov Model (HMM) profile of Hsp20 (PF00011) was downloaded from the Pfam protein family database (http://pfam.xfam.org/) (Finn et al. 2011) for the identification of Hsp20 proteins. The obtained high-quality protein set $\left(E\right.$-value $\left.<1 \times 10^{-10}\right)$ was aligned and used to construct a barley specific Hsp20 HMM using hmmbuild from the HMMER v3 suite. The new barley-specific HMM was used to select all proteins that were downloaded from the barley genome (Hordeum_vulgare.IBSC_v2) with an $E$-value $<0.01$. The selected barley Hsp20 sequences were matched to Hsp20 conserved domains (PF00011) using ClustalW (Larkin et al. 2007), then removed the incomplete sequences. The remaining Hsp20 protein sequences were submitted to Pfam (http://pfam.xfam.org/) and the Simple Modular Architecture Research Tool (SMART, http://smart.embl-heidelberg.de/) to confirm the conserved Hsp20 domain.

The chromosomal positions of the HvHsp20 genes were acquired from Ensembl database 113 (Hordeum_vulgare.Hv_IBSC_PGSB_v2.39). Each of these genes was mapped on chromosomes 114 using the Map Gene2chromosome (MG2C, version 2.0) tool (http://mg2c.iask.in/).

\section{Conserved motifs and transcript structures analysis}


The conserved motifs among subgroups of Hsp20 proteins were analyzed by the program of 117 Multiple Expectation for Motif Elicitation (MEME; version 4.10.0) with the following 118 parameters: maximum of 10 motifs, any number of repetitions, and an optimum motif width of 119 6-50 amino acid residues. Exon-intron structures of barley Hsp20 genes were identified on the 120 Gene Structure Display Server (GSDS, http://gsds.cbi.-pku.edu.cn/) (Hu et al. 2014).

\section{Phylogenetic analysis}

122 To illuminate evolutionary relationship of Hsp20s, the genomic sequence information of 123 Arabidopsis, rice, and sorghum was downloaded from the Ensembl Plants database 124 (http://plants.ensembl.org/index.html). All of the acquired sequences were aligned using

125

ClustalW (version 2.1) program with the default parameters (Larkin et al. 2007). The phylogenetic tree was constructed by MEGA X with bootstrap test of 1000 times, which was visualized in Figtree (http://tree.bio.ed.ac.uk/software/figtree/).

\section{Cis-acting regulatory element prediction in promoter regions}

The upstream sequences $(1.5 \mathrm{~kb})$ of HvHsp20 sequences were submitted to PlantCARE database (http://bioinformatics.psb.ugent.be/webtools/plantcare/html/) to computationally predict various regulatory elements based on positional matrices, consensus sequences, and individual sites on particular promoter sequences (Magali et al. 2002), such as abscisic acid (ABA)responsive elements, heat stress elements (HSEs), low-temp and salt stresses, TC-rich repeats, and $\mathrm{W}$ boxes, etc.

\section{Expression analysis of $\mathrm{HSP} 20$ genes under biotic and abiotic stresses}

The Illumina RNA-seq data (GSE117068, GSE101304, and GSE82134) were downloaded from NCBI Sequence Read Archive (SRA) database to study the expression patterns of HvHsp20 genes under biotic and abiotic stress conditions. Digital gene expression analysis of the identified 
139

140

141

142

143

144

145

146

147

148

149

150

151

152

153

154

155

156

157

158

159

160

161

HvHsp20 genes was investigated using Hisat2, Htseq, and DESeq2 (Wen 2017). Expression ratios of treated samples relative to control met the threshold criteria of $|\operatorname{logFC}|>2.0$ with a $P<$ 0.05 was considered as differently expressed.

\section{RESULTS}

\section{Identification of Hsp20 family members in barley}

To identify all the Hsp20 candidate members in the barley, we searched for sequences that contained the ACD domain using the HMMER with barley-specific HMM model of PF00011, and a total of $143 \mathrm{Hsp} 20 \mathrm{~s}$ were identified. After removing the repetitive and/or incomplete sequences, the rest of Hsp20s were submitted to Pfam (http://pfam.xfam.org/) and the Simple Modular Architecture Research Tool (SMART, http://smart.embl-heidelberg.de/) to confirm the ACD domain. Finally, 96 candidate Hsp20 protein sequences were confirmed in barley, were coded by 38 Hsp20 gene sequences. The protein and gene sequences of barley Hsp20s were shown in Table S1.

\section{Phylogenetic analysis of $\mathrm{HvHsp20}$ gene family}

To analyze the phylogenetic relationships of Hsp20 genes in barley, rice, sorghum, and Arabidopsis, a phylogenetic tree was constructed using full-length Hsp20 proteins (Fig. 1). All these Hsp20s were grouped into 7 distinct subfamilies based on their predicted subcellular localizations and the number of ACD domain, including $\mathrm{C}$ (cytosol), MT (mitochondria), CP (chloroplast), ER (endoplasmic reticulum), PX (peroxisome), and ACD subfamilies followed with 13 Hsp20s could not be classified into any subfamilies. In addition, the $\mathrm{C}$ subfamily was subdivided into 4 subgroups (CI-CIV) and MT subfamily was subdivided into 2 subgroups (MTI

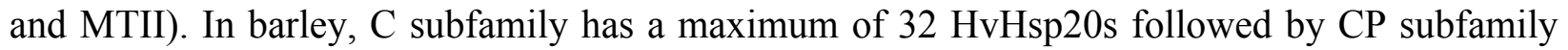


162 consisting of $25 \mathrm{HvHsp20s}$. Most of Hsp20s were classified into C subfamily, suggesting that

163 cytosol might be a main functional area for plant Hsp20s. We found an interesting result that CP

164 and MT subfamily members had a close relationship (Fig. 1), which is consistent with the

165 opinion that MT subfamily evolved later from the CP subfamily (Waters 2013).

\section{Gene structures and motifs of HvHsp20s}

167 The Gene Structure Display Server (GSDS) website was used to analyze the structural 168 characteristics of the Hsp20 genes in barley. The structural characteristic of $86 H v H s p 20$ genes that have normal intron length was showed in Fig. 2A. For another $10 H v H s p 20$ genes, the length of intron is much longer than that of extron, was shown in Fig. 2B. The number of introns in the $H v H s p 20$ genes varies from 0 to 10 , with $35 H v H s p 20$ genes (36\%) were intronless. About $45 \%$ (43) of $H v H s p 20$ genes contain one intron, and 18 (19\%) had two or more introns. One HvHsp20 gene contains 10 introns and 2 contain 6 introns (Fig. 2A and B). According to the number of introns, these $H v H s p 20$ genes were divided into three patterns: the first pattern has no intron, the second pattern has one intron, and the third pattern has more than one intron (Ouyang et al. 2009). Most of HvHsp20 genes $(81 \%, 78)$ belonged to the first and the second pattern, which was similar to the results reported on Hsp20 proteins in tomato (Yu et al. 2016).

Using the MEME tool, 10 types of consensus motifs in HvHsp20 proteins were identified (Fig. 3). The lengths of these conserved motifs varied from 15 to 50 amino acids (Motif logos shown in Fig. S1). The majority of the HvHsp20 proteins (50) contained Motif 1 while Motif 9 was found in only $5 \mathrm{HvHsp} 20$ proteins. The multiple sequence alignment analysis and sequence logo revealed that the ACD domain was formed by two conserved regions, conserved region I and conserved region II (Fig. 4), moreover, most of motif 1 and 6 located in consensus region I, and consensus region II contained motif 5, 7 and 8. Motif 3 and 9 located in the N-terminal of 
185 Hsp20, motif 10 located in C-terminal, while motif 4 and 2 located between consensus region I 186 and consensus region II of ACD domain (Table S2). Furthermore, HvHsp20 proteins in the same 187 subgroup present similar patterns of motif distribution, suggesting that these genes might have 188 relatively high conservation.

189

190

191

192 193

\section{Chromosomal location of $\mathrm{HvHsp} 20$ genes}

Among 38 predicted $H v H s p 20$ genes, 37 are randomly distributed across the 7 barley chromosomes, except for the HORVUOHrlG020420 which could not be located at anyone chromosome (Fig. 5). The distribution of the HvHsp20 genes on each chromosome is uneven. Some HvHsp20 genes are clustered on chromosomes 3 and 7, some are scattered on chromosomes 2 and 6, and most of these HvHsp20 genes were located at the distal ends of the chromosomes. The number of HvHsp20 genes on each chromosome is different. Chromosome 3 has the most number of HvHsp20 genes (10 genes), followed by chromosomes 4 (9 genes). Chromosomes 6 and 7 have the same number of HvHsp20 genes ( 4 genes). Chromosome 1 has 5 HvHsp20 genes, chromosome 5 has 3 genes, while only 2 genes on chromosome 2.

\section{Stress-related cis-elements in the HvHsp20 promoters}

To further explore the regulatory mechanisms of $H v H s p 20$ genes in response to stress conditions, the cis-elements in the promoter region (the $1.5 \mathrm{~kb}$ upstream sequences from the translation start sites) of the HvHsp20 genes were further analyzed. Nine stress response elements were analyzed and displayed in Fig. 6, including ABRE, DRE_core, G-Box, LTR, TCrich repeats, W-box, MBS, CCAAT-box, and MYB. Among these 38 genes, except for 2 HvHsp20 genes (HORVU6Hr1G015130 and HORVU7Hr1G036470), the other possessed at least 1 type of stress-response-related cis-element; and HORVU1Hr1G035950, had 8 categories cis-elements, indicating that the expressions of HvHsp20 genes were related to various stress 
208 responses. In total, one or more G-Box existed in $29 H v H s p 20 s$ (76\%), followed by ABRE which 209 was found in $28 H v H s p 20 s$ (74\%). In addition, one or two TC-rich repeats existed in 12 $210 H v H s p 20 s$, while DRE_core, W-box, LTR, MBS, CCAAT-box, and MYB were presented in 20,

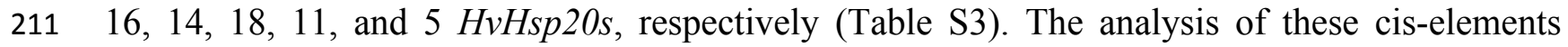
212 suggested that $H v H s p 20$ genes could response to different stress conditions.

\section{Expression profiles of $\mathrm{HvHsp20}$ genes under biotic and abiotic stresses}

214 To explore the responses of $H v H s p 20$ genes to various stresses, expression patterns of HvHsp20 genes in response to abiotic stresses (arsenate treatment and heat) and biotic stress 216 (powdery mildew infection) were investigated using RNA-seq data. Overall, the 37 out of the

217 218

219

220

221

222

223

224

225

226

227 228

229 230

identified $38 \mathrm{HvHsp20}$ genes showed differential expression patterns under these conditions (Fig. 7). Arsenate stress induced relatively more fluctuations in the transcript abundance of HvHsp20 than that of the heat stress and powdery mildew infection. A total of $7 H v H s p 20$ genes were induced in response to arsenate stress in arsenate sensitive barley genotype (ZDB475), and 19 genes increased in arsenate and phosphate stress (Fig. 7B). In arsenate tolerant barley genotype (ZDB160), $19 H v H s p 20$ genes showed up-regulated expression under both arsenate stress and arsenate and phosphate stress (Fig. 7A). Some of increased expression in HvHsp20 genes induced by arsenate stress displayed a slight declined expression trends in arsenate and phosphate stress. In addition, more up-regulated HvHsp20 genes in ZDB160 than in ZDB475 implied $H s p 20 s$ played critical roles in response to arsenate stress. Over $60 \%$ of $H v H s p 20$ genes were up-regulated under abiotic stresses, which up to $90 \%$ in heat stress (Fig. 7C and D), while for powdery mildew stress, more than $80 \%$ of HvHsp20 genes were down-regulated (Fig. 7E). In addition, three HvHsp20 genes, HORVU7Hr1G036540, HORVU7Hr1G036470, and HORVU3Hr1G007500, were up-regulated under biotic and abiotic stresses (Fig. 7), suggesting 
231 they have major functions in response to various stress conditions. In total $10 H v H s p 20$ genes

232 showed differential expression trends in all of the stress conditions, three HvHsp20 genes

233 (HORVU3Hr1G006930, HORVU4Hr1G072700, and HORVU6Hr1G070230) showed specific-

234 expression under biotic stress (Fig. S2).

235

\section{DISCUSSION}

237 Hsp20 proteins play vital roles in plant growth and development processes as well as biotic

238

239

240

241

242

243

244

245

246

247

248

249

250

251

252

253

and abiotic stress responses. With the availability of the whole genome sequence, the Hsp20 family genes have been identified in many plants, such as Arabidopsis and rice (Scharf et al. 2001; Sarka et al. 2009). Preliminary analysis of the barley Hsp20 gene family has been performed by Reddy et al. (2014). Benefiting from the high-quality reference genome information of barley (Mascher et al. 2017), by constructing barley-specific HMM, we identified $38 H v H s p 20$ genes coding $96 \mathrm{HvHsp} 20$ proteins. The number of identified HvHsp20 genes was more than Reddy et al. (2014) reported, which might be because we took different identification methods and (or) more Hsp20 genes were annotated recently. Then we analyzed their structure, chromosomal location, phylogeny, and expression pattern diversity with respect to biotic and abiotic stresses, which provides useful information for understanding the $H v H s p 20$ gene family and will establish a basis for future analyzing functional divergence of the Hsp20 genes in barley. The number of identified Hsp20 genes in graminaceous plants, such as rice and wheat, was 39 and 163, respectively (Ouyang et al., 2009, Muthusamy et al., 2017). The number of members in barley was 38 , which close to in rice, but was fewer than in wheat, suggesting the possibility of a gene gain event from diploid to hexaploid during the evolutionary process. Previously, plants Hsp20 family members were classified into 5 subfamilies according to their subcellular 
254 localization including C, CP, MT, ER, and PX subfamilies, and ACD subfamily (Scharf et al., 255 2001; Siddique et al., 2008). In our study, to reveal phylogenetic relationship of Hsp20 family 256 members, Hsp20s from Arabidopsis, rice, sorghum, and barley, were used to construct a 257 phylogenetic tree (Fig. 1). The results showed that these Hsp20s existed in 7 subfamilies 258 including some Hsp20s could not be grouped. Interestingly, we found that 25 barley Hsp20s 259 belonged to CP subfamily, which was much more than the number of Hsp20s in rice and wheat 260 (Ouyang et al., 2009; Muthusamy et al., 2017), suggesting barley Hsp20s might had undergone 261 duplication and/or recombination events during evolution.

262 The exon/intron structure plays a vital role in organismal evolution (Guixia et al. 2012).

263 Here we found the positions, sizes, and sequences of the introns were quite different between the 264 identified HvHsp20 genes (Fig. 2). About 36\% HvHsp20 genes do not have introns, which is 265 slightly lower than in rice genes predicted to be intronless (48.72\%) (Ouyang et al. 2009), most 266 of these HvHsp20 genes belonged to CI and CII subfamilies. Many members of the CP and MTI 267 subfamilies had only one intron, and the length of introns is relatively short. This is consistent with the report that plants tend to retain the genes with no intron or a short intron (Mattick and 269 Gagen 2001). To response to environmental challenges, fewer introns in genes are benefit to be rapidly activated (Jeffares et al. 2008). Hsp20 genes are one of the rapidly expressed genes under 271 a variety of stresses (Sarkar et al. 2009; Yu et al. 2016). The absence of introns or their presence 272 with smaller size may in accordance with the needs of the rapid induction of Hsp20 genes. In this 273 study, we found that most of the HvHsp20 genes with one or no intron were induced under 274 several stresses, but genes with many introns, such as HORVU6Hr1G070230, were induced 275 during only powdery mildew infection. Furthermore, the majority members in the same 276 phylogenetic subfamily had similar motif compositions (Fig. 3). This correlation between intron 
277 numbers and motif arrangement supported the previous classification of the HvHsp20 genes.

278 These results may provide facilitation to identify the functions of HvHsp20 genes and further to 279 discover their function in responses to environmental stresses.

280 From the expression analysis, it has been demonstrated that Hsp20 genes play important

281

282

283

284

285

286

287

288

289

290

291

292

293

294

295

296

297

298

299 roles in the control of plants in response to diverse environmental challenges (Waters 2013). In this study, we investigated the expression profiles of the HvHsp20 genes in barley after biotic and abiotic stress treatments (Fig. 7). The data demonstrated that numerous $H v H s p 20$ genes were significantly induced to a larger extent under abiotic stresses including arsenic and heat stress, and 21 Hsp20 genes showed differential expression patterns under two stress treatments, which belonged to CI and CII subfamilies, confirming the protective role of $H v H s p 20$ family members in barley. For heat stress, among differential expressed $H v H s p 20$ genes, the vast majority of them grouped in CI, CII, and CP subfamilies were up-regulated both in barley shoots and roots. Previous research showed that the CI and CII subfamilies both have chaperone activity, and the CI proteins are lower efficiency than CII members in preventing irreversible aggregation (Eman et al. 2010). However, by knockdown the CI and CII genes, other studies suggested that the CI sHSPs have thermoprotective roles but the CII sHSPs do not (Markus et al. 2004; Tripp 2010). The biochemical and biological differences between the subfamilies may be consistent with the patterns of N-terminal sequence conservation within subfamilies (Waters 2013). Continued studies of the relationship between sequence differences and functional changes of $H s p 20$ genes will be crucial to further understanding the function of sHSPs. Furthermore, we found that the arsenic stress inducibility of HvHsp20 genes in tolerant plants was stronger than that in susceptible plants, suggesting that HvHsp20 genes play vital roles in response to heavy metal stress.

Peer) reviewing PDF | (2018:12:33715:1:2:NEW 12 Mar 2019) 

virus, bacteria and fungus (Park and Seo 2015). In the present study, five HvHsp20 genes

302 303

showed an increased expression during the first few hours of powdery mildew infection, then the expression dropped rapidly following the infection. The results are consistent with the report that biotic stress can induce the gene expression of some but not all sHSPs (Siddique et al. 2008), suggesting that HvHsp20 genes may confer biotic stress tolerance in barley. Among these 5 HvHsp20 genes, three genes (HORVU7Hr1G036540, HORVU7Hr1G036470, and HORVU3Hr1G007500) were also significantly induced under abiotic stresses including arsenic and heat stress, suggesting these $3 H v H s p 20$ genes might play important roles in mediating the response of barley plants to various stresses. However, further analysis is needed to investigate how the $3 H v H s p 20$ genes influence plant defense response.

\section{CONCLUSIONS}

In the current study, a genome-wide analysis of barley HvHsp20 genes family was performed, and 38 putative HvHsp20 genes were identified. A comprehensive analyse of HvHsp20 genes on gene structures, chromosomal location, phylogenetic relationship stressrelated cis-elements, and expression patterns under biotic and abiotic stresses, were conducted by using bioinformatics and RNA-seq data. Most of HvHsp20 genes belonged to CI, CII, and CP subfamilies showed differential expression under stress conditions, indicating that HvHsp20 genes play important roles in response to stress. This study provided comprehensive information on the HvHsp20 gene family in barley and will aid in investigating the function of $H v H s p 20$ genes. 
323

324

325

326

327

328

329

330

331

332

333

334

335

336

337

338

339

340

341

342

343

344

345

\section{REFERENCES}

Bondino HG, Valle EM, Have AT. 2012. Evolution and functional diversification of the small heat shock protein/ $\alpha$-crystallin family in higher plants. Planta 235(6):1299-1313 DOI $10.1007 / \mathrm{s} 00425-011-1575-9$.

Eman B, Christopher J, Vicki W, Elizabeth V. 2010. Mechanistic differences between two conserved classes of small heat shock proteins found in the plant cytosol. Journal of Biological Chemistry 285(15):11489-11497 DOI 10.1074/jbc.M109.074088.

Eyles SJ, Gierasch LM. 2010. Nature's molecular sponges: small heat shock proteins grow into their chaperone roles. Proceedings of the National Academy of Sciences of the United States of America 107(7):2727-2728 DOI 10.1073/pnas.0915160107.

Finn RD, Clements J, Eddy SR. 2011. HMMER web server: interactive sequence similarity searching. Nucleic Acids Research (Web Server issue) 39:29-37 DOI 10.1093/nar/gkr367.

Giese KC, Vierling E. 2004. Mutants in a small heat shock protein that affect the oligomeric state. Analysis and allele-specific suppression. Journal of Biological Chemistry 279(31):32674-32683 DOI 10.1074/jbc.M404455200.

Guixia X, Chunce G, Hongyan S, Hongzhi K. 2012. Divergence of duplicate genes in exonintron structure. Proceedings of the National Academy of Sciences of the United States of America 109(4):1187-1192 DOI 10.1073/pnas.1109047109.

Guo M, Liu JH, Lu JP, Zhai YF, Wang H, Gong ZH, Wang SB, Liu MH. 2015. Genomewide analysis of the CaHsp20 gene family in pepper: comprehensive sequence and expression profile analysis under heat stress. Frontiers in Plant Science 6:806 DOI10.3389/fpls.2015.00806.

Hu B, Jin J, Guo AY, Zhang H, Luo J, Gao G. 2014. GSDS 2.0: an upgraded gene feature 
346

347

visualization

server.

Bioinformatics

31(8):1296-1297

DOI 10.1093/bioinformatics/btu817.

Jaya N, Garcia V, Vierling E. 2009. Substrate binding site flexibility of the small heat shock protein molecular chaperones. Proceedings of the National Academy of Sciences of the United States of America 106(37):15604-15609 DOI 10.1073/pnas.0902177106.

Jeffares DC, Penkett CJ, Bähler J. 2008. Rapidly regulated genes are intron poor. Trends in genetics 24(8):375-378 DOI 10.1016/j.tig.2008.05.006.Larkin MA, Blackshields G, Brown NP, Chenna R, Mcgettigan PA, Mcwilliam H, Valentin F, Wallace IM, Wilm A, Lopez R. 2007. Clustal W and Clustal X version 2.0. Bioinformatics 23(21):29472948 DOI 10.1093/bioinformatics/btm404.

Lopescaitar VS, Carvalho MCD, Darben LM, Kuwahara MK, Nepomuceno AL, Dias WP, Abdelnoor RV, Marcelinoguimarães FC. 2013. Genome-wide analysis of the Hsp20 gene family in soybean: comprehensive sequence, genomic organization and expression profile analysis under abiotic and biotic stresses. BMC Genomics 14(1):577 DOI $10.1186 / 1471-2164-14-577$.

Lozano R, Hamblin MT, Prochnik S, Jannink JL. 2015. Identification and distribution of the NBS-LRR gene family in the Cassava genome. BMC Genomics 16(1):360 DOI 10.1186/s12864-015-1554-9.

Magali L, Patrice D, Gert T, Kathleen M, Yves M, Yves VDP, Pierre R, Stephane R. 2002. PlantCARE, a database of plant cis-acting regulatory elements and a portal to tools for in silico analysis of promoter sequences. Nucleic Acids Research 30(1):325-327 DOI 10.1093/nar/30.1.325.

Markus P, Joanna T, Dirk Z, Christian W, Dirk H, Sybille W, Daniela B, Klaus-Dieter S. 
2004. Role of Hsp17.4-CII as coregulator and cytoplasmic retention factor of tomato heat stress transcription factor HsfA2. Plant Physiology 135(3):1457-1470 DOI $10.2307 / 4356505$.

Mascher M, Gundlach H, Himmelbach A, Beier S, Twardziok SO, Wicker T, Radchuk V, Dockter C, Hedley PE, Russell J. 2017. A chromosome conformation capture ordered sequence of the barley genome. Nature 544(7651):427-433 DOI 10.1038/nature22043.

Mattick JS, Gagen MJ. 2001. The evolution of controlled multitasked gene networks: the role of introns and other noncoding RNAs in the development of complex organisms. $\begin{array}{llll}\text { Molecular Biology } & \& & \text { Evolution } & \text { 18(9):1611-1630 }\end{array}$ DOI:10.1093/oxfordjournals.molbev.a003951.

Muthusamy SK, Dalal M, Chinnusamy V, Bansal KC. 2017. Genome-wide identification and analysis of biotic and abiotic stress regulation of small heat shock protein (HSP20) family genes in bread wheat. Journal of Plant Physiology 211:100-113 DOI 10.1016/j.jplph.2017.01.004.

Ouyang Y, Chen J, Xie W, Wang L, Zhang Q. 2009. Comprehensive sequence and expression profile analysis of Hsp20 gene family in rice. Plant Molecular Biology 70(3):341-357 DOI 10.1007/s11103-009-9477-y.

Park CJ, Seo YS. 2015. Heat shock proteins: A review of the molecular chaperones for plant immunity. Plant Pathology Journal 31(4):323-333 DOI 10.5423/ppj.rw.08.2015.0150.

\section{Reddy PS, Kishor PB, Christiane S, Markus K, Lennart EL, Justin L, Reddy MK, Nese S.} 2014. Unraveling regulation of the small heat shock proteins by the heat shock factor $H v H s f B 2 c$ in barley: its implications in drought stress response and seed development. PLOS One 9(3):e89125 DOI 10.1371/journal.pone.0089125. 
392 Sarkar NK, Kim YK, Grover A. 2009. Rice sHsp genes: genomic organization and expression

393

394

395

396

397

398

399

400

401

402

403

404

405

406

407

408

409

410

411

412

413

414 profiling under stress and development. BMC Genomics 10(1):393 DOI 10.1186/14712164-10-393.

Scharf KD, Siddique M, Vierling E. 2001. The expanding family of Arabidopsis thaliana small heat stress proteins and a new family of proteins containing $\alpha$-crystallin domains (Acd proteins). Cell Stress Chaperones 6(3):225-237 DOI 10.1379/14661268(2001)0062.0.CO;2.

Shoseyov O. 2004. Role of plant heat-shock proteins and molecular chaperones in the abiotic stress response. Trends in Plant Science 9(5):244-252 DOI 10.1016/j.tplants.2004.03.006.

Siddique M, Gernhard S, Koskull-Döring PV, Vierling E, Scharf KD. 2008. The plant sHSP superfamily: five new members in Arabidopsis thaliana with unexpected properties. Cell Stress Chaperones 13(2):183-197 DOI 10.1007/s12192-008-0032-6.

Sun W, Montagu MV, Verbruggen N. 2002. Small heat shock proteins and stress tolerance in plants. Biochimica et Biophysica Acta- Gene Structure and Expression 1577:1-9 DOI 10.1016/S0167-4781(02)00417-7.

Tripp J. 2010. Functional dissection of the cytosolic chaperone network in tomato mesophyll protoplasts. Plant Cell Environment 32(2):123-133 DOI 10.1111/j.13653040.2008.01902.x.

Waters ER. 2013. The evolution, function, structure, and expression of the plant sHSPs. Journal of Experimental Botany 64(2):391-403 DOI 10.1093/jxb/ers355.

Wen G. 2017. A simple process of RNA-Sequence analyses by Hisat2, Htseq and DESeq2. International Conference Pp:11-15 DOI: 10.1145/3143344.3143354.

Yu J, Cheng Y, Feng K, Ruan M, Ye Q, Wang R, Li Z, Zhou G, Yao Z, Yang Y, 2016. 
415

416

417

418

419

420
Genome-wide identification and expression profiling of tomato Hsp20 gene family in response to biotic and abiotic stresses. Frontiers in Plant Science 7:806 DOI 10.3389/fpls.2016.01215.

Yuko N, Shunji S. 2013. Environmental stresses induce misfolded protein aggregation in plant cells in a microtubule-dependent manner. International Journal of Molecular Sciences 14(4):7771-7783 DOI 10.3390/ijms 14047771. 
Figure 1 (on next page)

Figure 1 Phylogenetic relationship of Hsp20s from Arabidopsis, rice, sorghum, and barley.

The phylogenetic tree was constructed using MEGA X with 1000 bootstrap replications. 


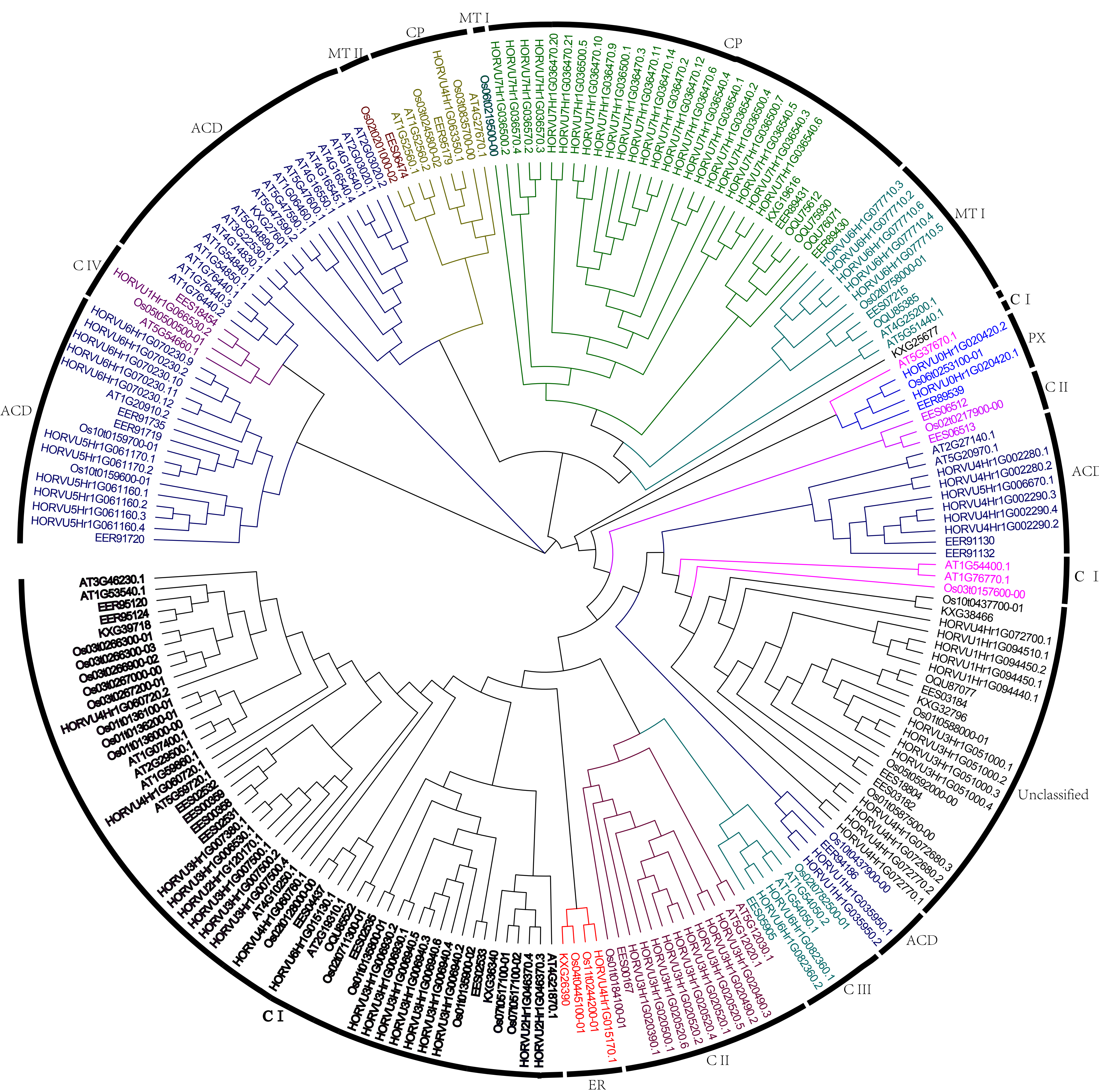


Figure 2

Figure 2 Exon/intron structures of barley HvHsp20 genes.

(A) The gene structures of $86 \mathrm{HvHsp20}$ that have normal intron length. (B) The structural characteristic of $10 \mathrm{HvHsp20}$ genes that possess too longer introns. Boxes filled with blue represent exons, solid black lines represent introns. Scale at bottom is in kb. 


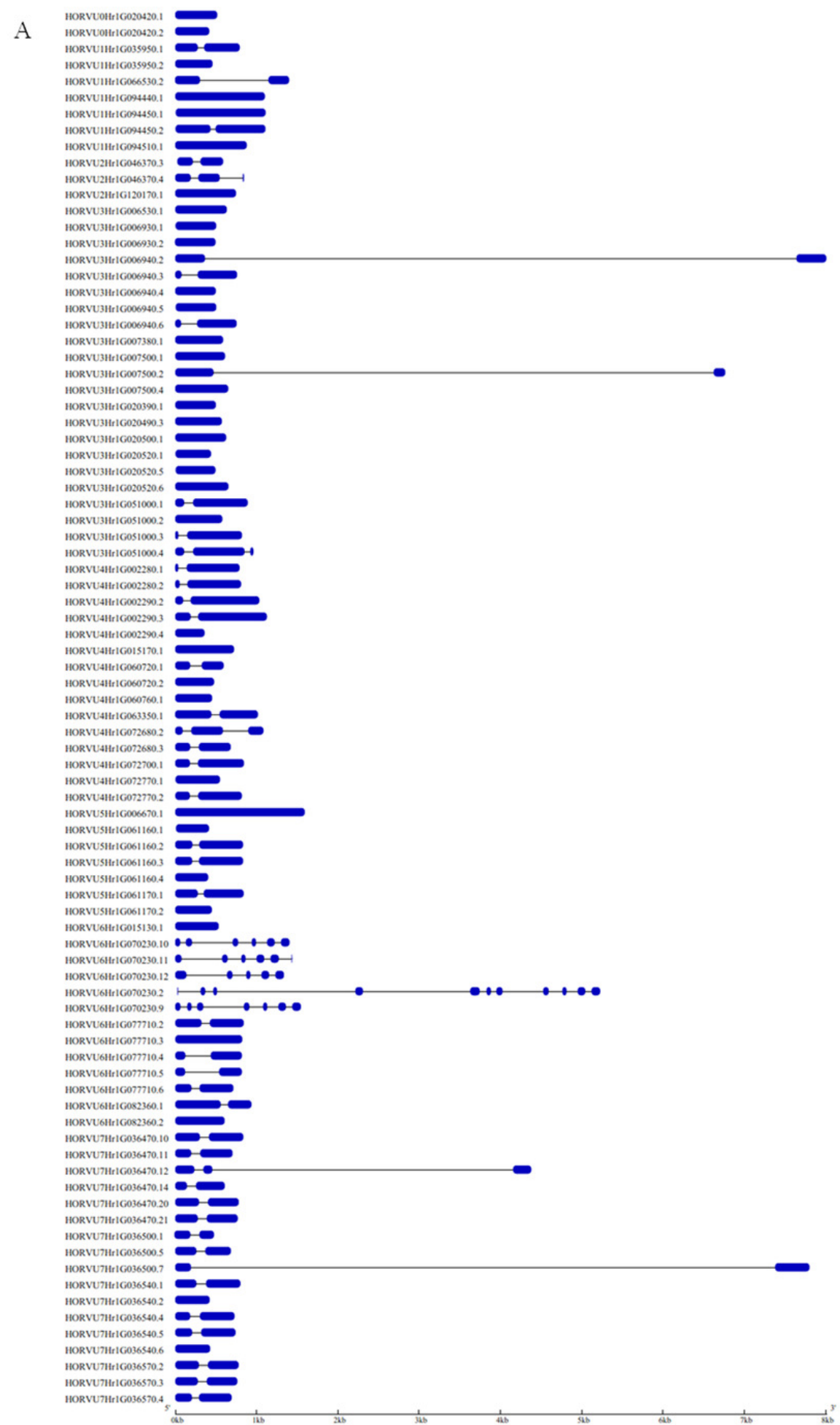

$$
\text { Legend: }
$$

В нов

HORVU3HrIG020520.2

HORVU3HrIG020520.4

HORVU7H+IG036470.2

HORVU7HrIG036470.3

HORVU7HrIG036470.6

HORVU7HrIG036470.9

HORVU7HrIG036500.4

HORVU7HrIG036540.3

$$
\text { Legend: - Intron }
$$

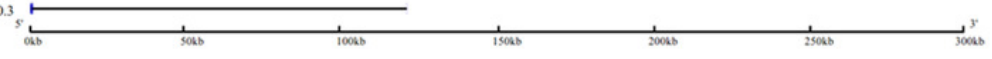

Peer] reviewing PDF | (2018:12:33715:1:2:NEW 12 Mar 2019) 
Figure 3 (on next page)

Figure 3 Phylogenetic relationships and motif compositions of HvHsp20 proteins.

(A) Multiple alignment of Hsp20 domain was executed using ClustalW and the phylogenetic tree was constructed with MEGA X using full-length amino acid sequences of identified Hsp20 proteins in barley. (B) Distributions of conserved motifs in HvHsp20 proteins. Each colored box represents a motif in the protein. 
C HORVU2Hr1G046370.3 - - - -

HORVU2Hr1G046370.4

HORVU6Hr1G070230.9 _ _ _ _ _ _

HORVU6Hr1G070230.2

- HORVU6Hr1G070230.10

[ HORVU6Hr1G070230.12

HORVU6Hr1G070230.11

$[$ HORVU4Hr1G002290.4

L HORVU4Hr1G002290.2

- HORVU5Hr1G006670.1

[ HORVU1Hr1G035950.1

HORVU1Hr1G035950.2

HORVU4Hr1G002280.1

Pe HORVU4Hr1G002280.2 -

4ORVU4Hr1G072770.2

[ HORVU4Hr1G072770.1

- HORVU4Hr1G072700.1

[ HORVU4Hr1G072680.3

HORVU4Hr1G072680.2

HORVU1Hr1G094510.1

HORVU1Hr1G094440.1

- HORVU1Hr1G094450.2

- HORVU3Hr1G051000.1

[ HORVU3Hr1G051000.2 -

L HORVU3Hr1G051000.4

HORVU3Hr1G051000.3 - - -

HORVUOHr1G020420.1

HORVUOHr1G020420.2

HORVU3Hr1G006930.2

HORVU3Hr1G006930.1

HORVU3Hr1G006940.2

HORVU3Hr1G006940.4

- HORVU3Hr1G006940.6

HORVU3Hr1G006940.3

HORVU3Hr1G006940.5

HORVU1Hr1G066530.2

HORVU6Hr1G082360.1

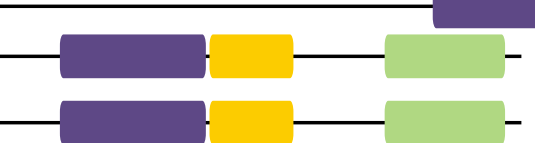

$-\square$

$+-$

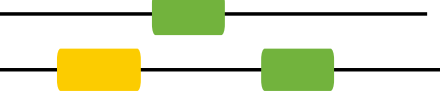

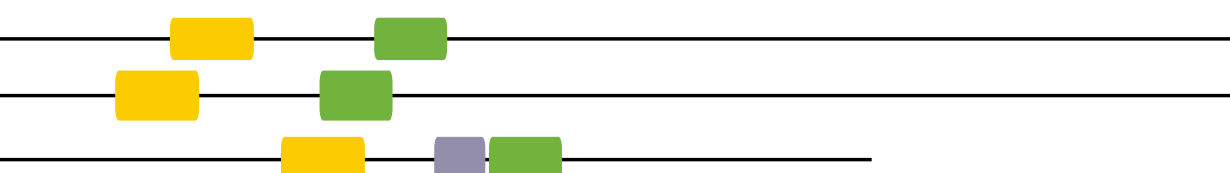

$+r+5$

$$
-
$$

- HORVU6Hr1G082

- HORVU3Hr1G020500.1

- HORVU3Hr1G020520.5

- HORVU3Hr1G020520.1

[ HORVU3Hr1G020520.2

HORVU3Hr1G020520.4

- HORVU3Hr1G020520.6

- HORVU3Hr1G020390.1

[ HORVU3Hr1G020490.3

[ HORVU3Hr1G020490.2

- HORVU4Hr1G060760.1

[ HORVU4Hr1G060720.2

HORVU4Hr1G060720.1

HORVU6Hr1G015130.1

HORVU4Hr1G015170.1 -

— HORVU3Hr1G007500.1

HORVU3Hr1G007500.2

HORVU3Hr1G007500.4

- HORVU2Hr1G120170.1

[ HORVU3Hr1G006530.1

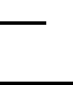

HORVU4Hr1G063350.1

HORVU5Hr1G061160.

$\left[\begin{array}{l}\text { HORVU5Hr1G061160.1 } \\ \text { HORVU5Hr1G061160.4 }\end{array}\right.$

L HORVU5Hr1G061160.3

[ HORVU5Hr1G061170.2.

- HORVU6Hr1G077710.2

[ HORVU6Hr1G077710.4

HORVU6Hr1G077710.6

HORVU6Hr1G077710.5

HORVU6Hr1G077710.3

HORVU7Hr1G036470.12

HORVU7Hr1G036540.4

HORVU7Hr1G036540.1

HORVU7Hr1G036540.2

HORVU7Hr1G036500.4

HORVU7Hr1G036470.2

HORVU7Hr1G036470.11

HORVU7Hr1G036470.14

HORVU7Hr1G036470.10

HORVU7Hr1G036470.9 -
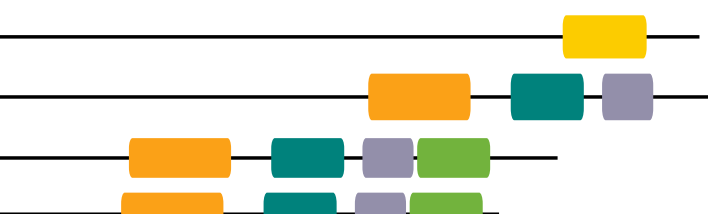

L- $-2+2$

- - - - -

$-2-$

- $2-1-$

- - - -

-

- - - - - - -

-

$+2-2$

-

$-2-2-$

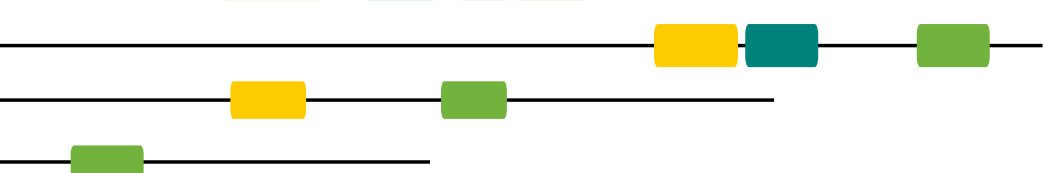

$\longrightarrow$

$+2$

-

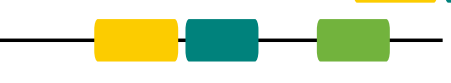

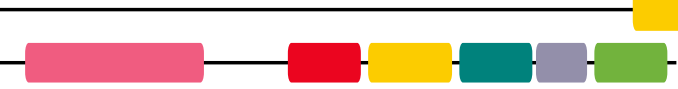

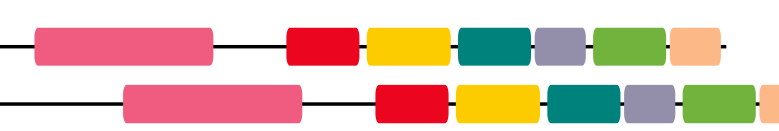

trat.

-

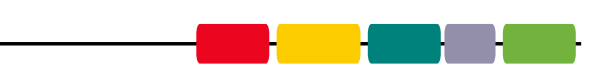

- $-2+1-$

_-

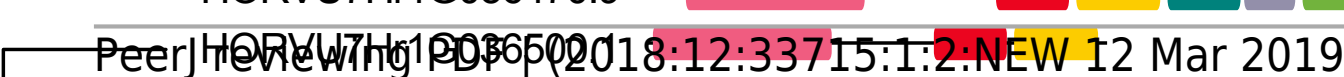

HORVU7Hr1G036470.3

HORVU7Hr1G036500.2

HORVU7Hr1G036500.7 .

HORVU7Hr1G036570.4 .

HORVU7Hr1G036570.2 -

- HORVU7Hr1G036570.2 ___ _ _

- HORVU7Hr1G036570.3 _ _ _ _ _

- HORVU7Hr1G036540.5

HORVU7Hr1G036540.3 —__ _ ? ?

HORVU7Hr1G036540.6 _

HORVU7Hr1G036470.20 _ _ _ _ _ _ _ _

$[$ HORVU7Hr1G036470.21 _ _ [ ]

L HORVU7Hr1G036470.6 __ _ _ _ _

HORVU7Hr1G036500.5 _ _ _ _ _ _

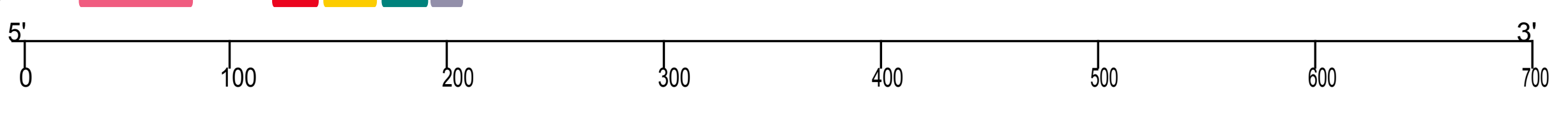


Figure 4 (on next page)

Figure 4 Multiple sequence alignment of ACDs of HvHsp20s.

Names of all members are listed on the left side of the figure. Conserved amino acid residues are indicated by gray background. Two conserved regions (conserved region I and conserved region II) are underlined at the bottom. 


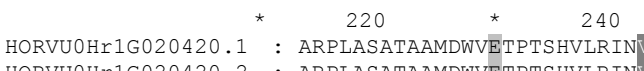
HORVUOHr1G020420.2 HORVU7H H $1 \mathrm{G} 036500$. HORVU7Hr1G036500.7
HORVU7Hr1G036500. HORVU7Hr1G036500.1 HORVU7Hr1G036570. HORVU7Hr1G036570.2
HORVU7Hr1G036570.3 HORVU7Hr1G036570.3
HORVU2Hr1G046370.3 HORVU1Hr1G094510. HORVU5Hr1G061160.3 HORVU5Hr1G061160. HORVU5Hr1G061160.4 HORVU5Hr1G061170.2 HORVUSHr1G061170. HORVU6Hr1G015130. HORVU4Hr1G015170. HORVU6Hr1G070230. HORVU 6 H $r 16070230.2$ HORVU6Hr1G070230.12 HORVU6Hr1G070230. HORVU6Hr1G082360. HORVU6HrIG082360.12 HORVU4H H 1 G072700.1 HORVU 4 Hr1G072680.2 HORVU4Hr1G060720.2 HORVU6Hr1G077710. HORVU6Hr1G077710. HORVU6Hr1G077710.5 HORVU6Hr1G077710.3 HORVU7Hr1G036470. HORVU7Hr1G036470.9. HORVU7Hr1G036470.20 HORVU7Hr1G036470.11: HORVU7Hr1G036470. HORVU7Hr1G036470.
HORVU7Hr1G036470. HORVU7Hr1G036470.2 HORV Hr1G036540.4 HORVU7H 1 1G036540.1 HORVU7Hr1G036540.2 HORVUTHr1G036540.3. HORVU7HrIG036540. HORVU1 Hr1G035950.1 HORVU1Hr1G035950.2 HORVU1 Hr1G094450.1 HORVU1 Hr1G094440. HORVU3Hr1G020390. HORVU3Hr1G007500.4 HORVU3Hr1G007500. HORVU3Hr1G020520.5 HORVU3Hr1G020520.

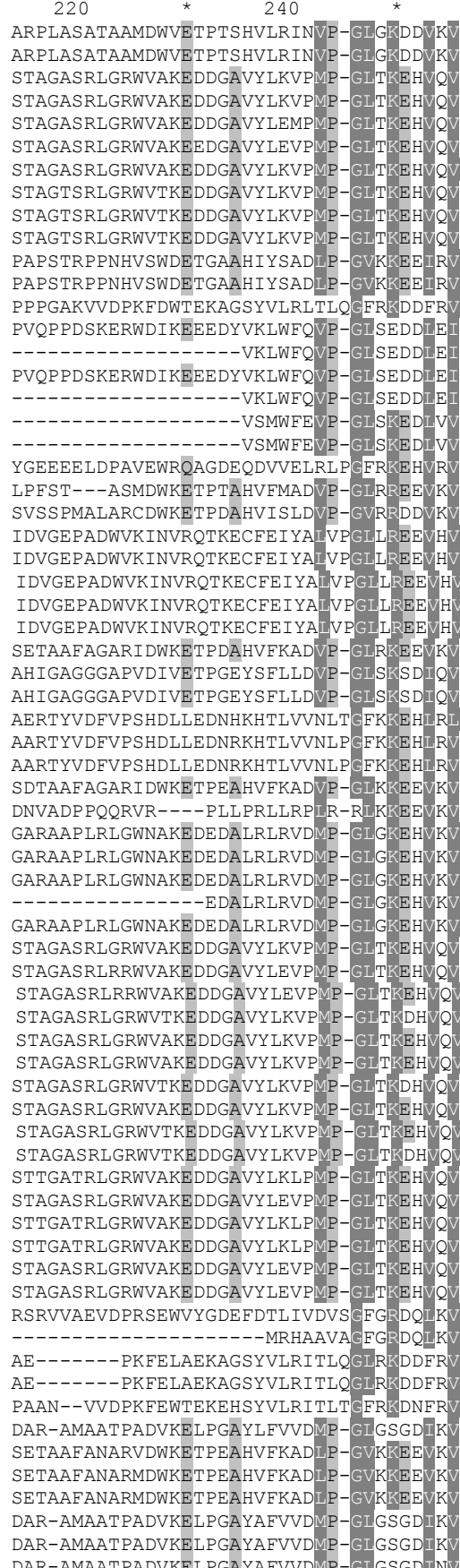

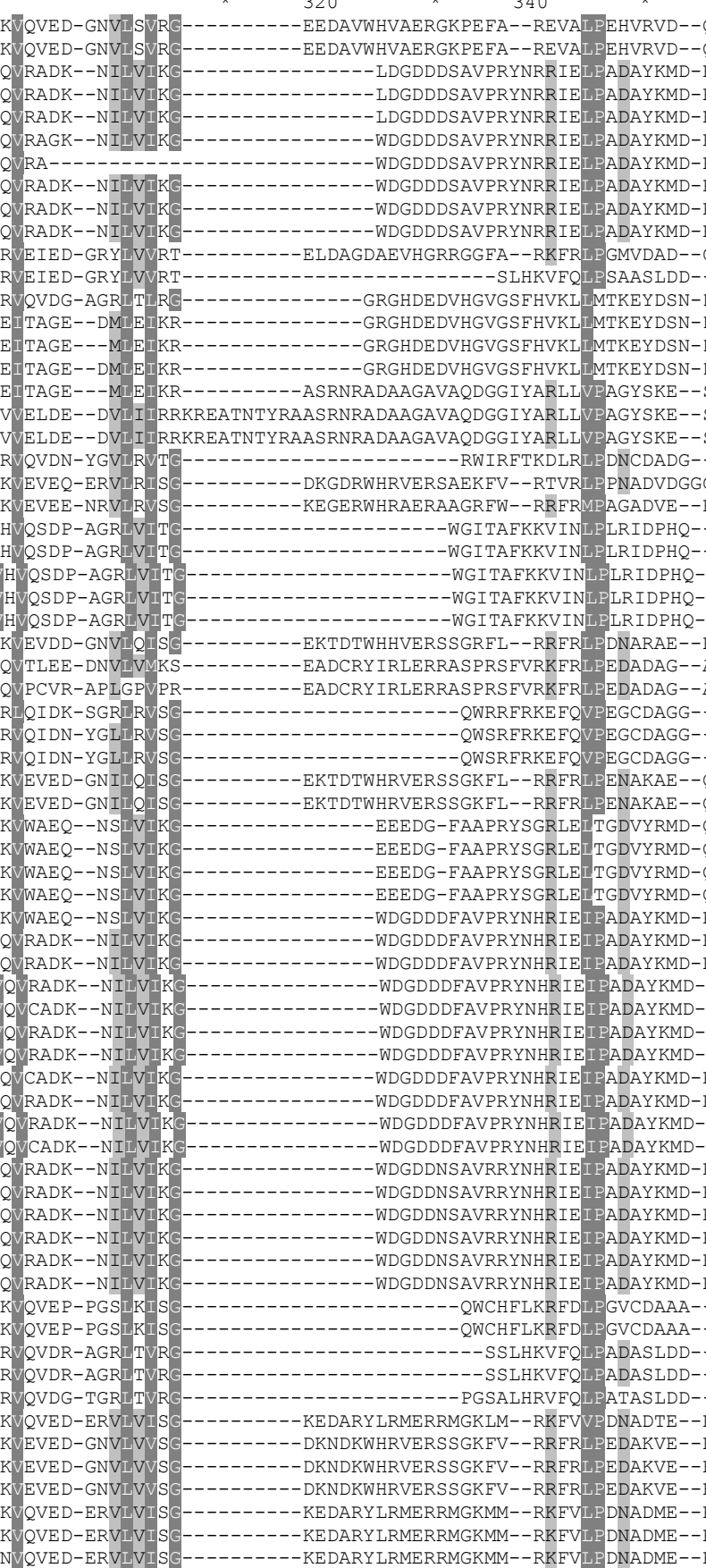

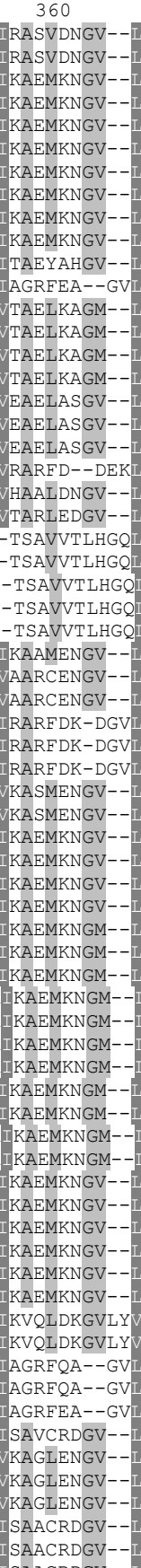
${ }^{*}$ Peer) reviewing PDF | (2018:12:33715:1:2:NEW 12 Mar 2019) 


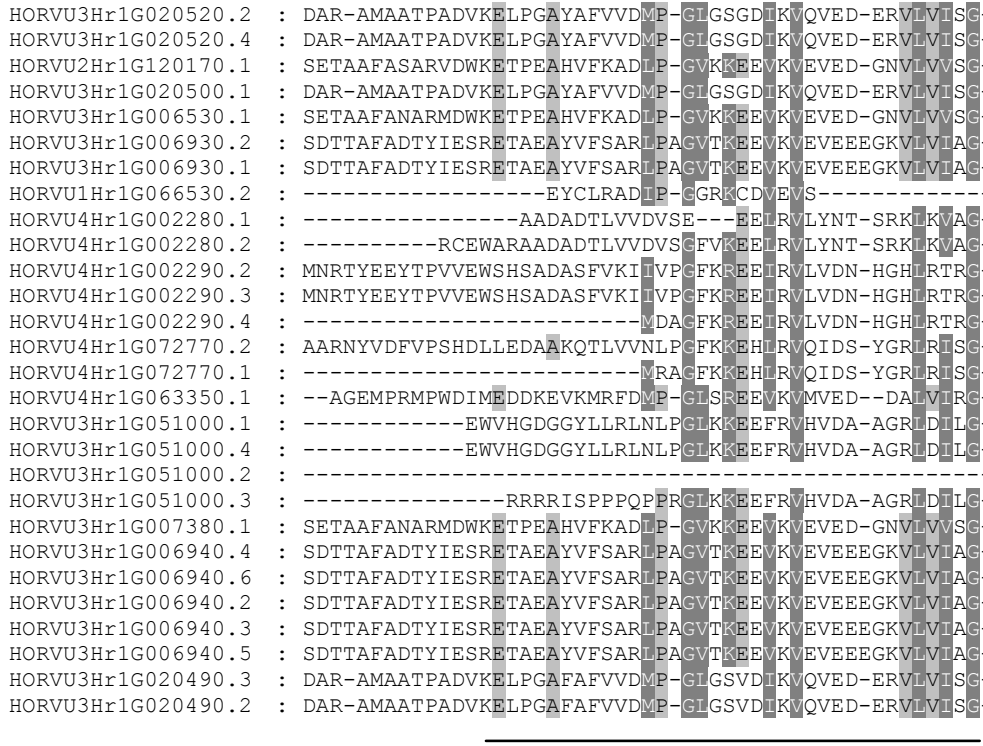

Conserved region I

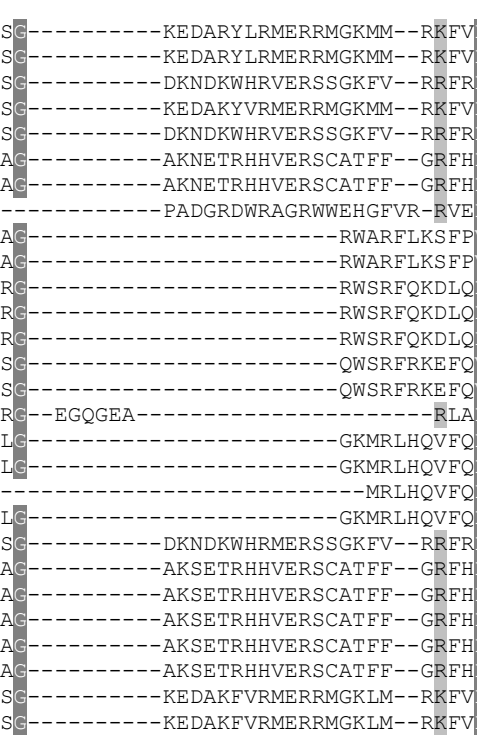
-KEDAKFVRMERRMGKLM--

RKFVLPENADME-

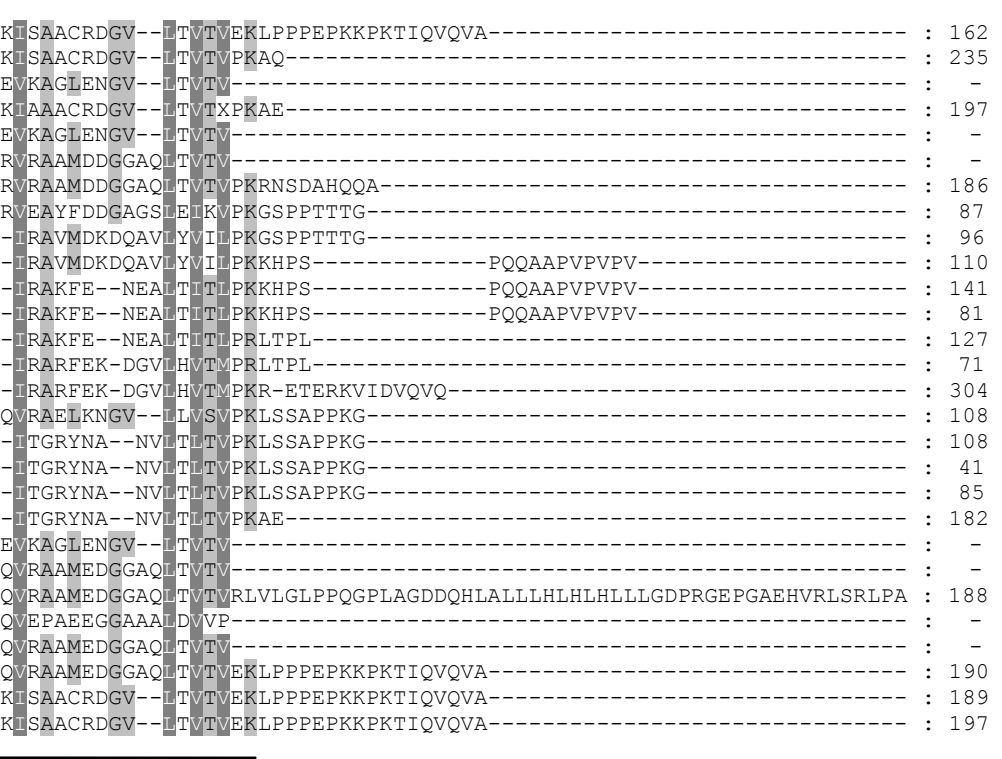

Conserved region II 
Figure $\mathbf{5}$ (on next page)

Figure 5 Chromosome localizations of barley HvHsp20 genes .

Chromosome numbers are shown on top of each bar. Scale on left is in Mb. 


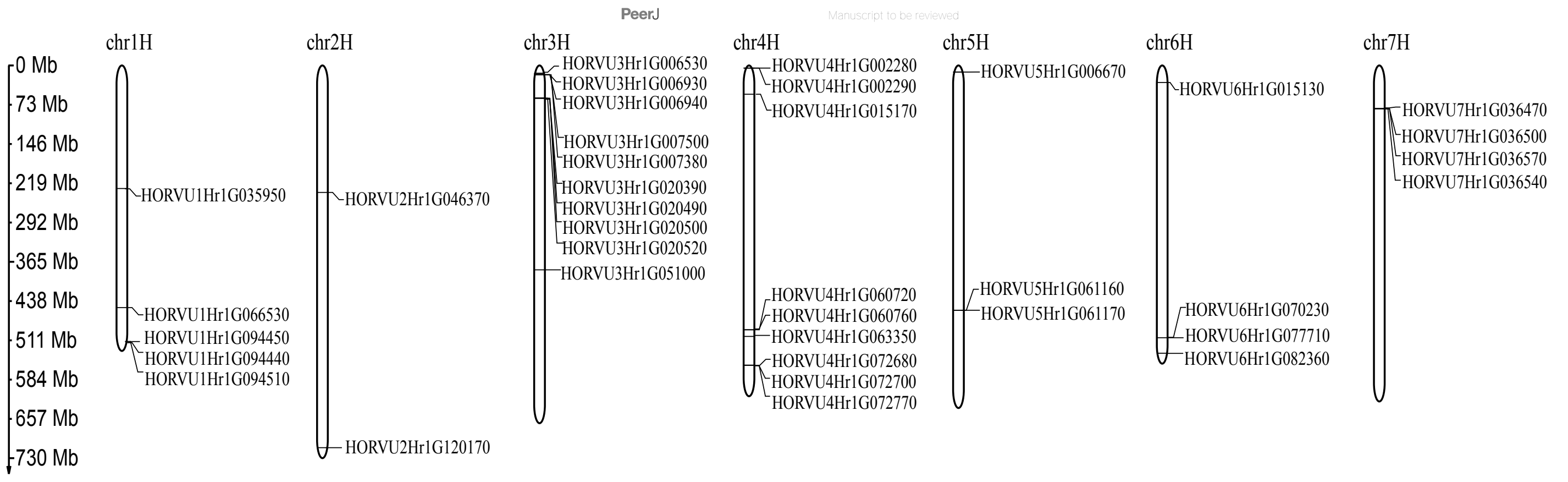


Figure 6 (on next page)

Figure 6 Predicted cis-elements in HvHsp20 promoter region.

Promoter sequences (-1.5kb) are analyzed by PlantCARE. 
HORVU0Hr1G020420 HORVU1Hr1G035950 HORVU1Hr1G066530 HORVU1Hr1G094440 HORVU1Hr1G094450 HORVU1Hr1G094510 HORVU2Hr1G046370 HORVU2Hr1G058170 HORVU3Hr1G006530 HORVU3Hr1G006930 HORVU3Hr1G006940 HORVU3Hr1G007380 HORVU3Hr1G007500 HORVU3Hr1G020390 HORVU3Hr1G020490 HORVU3Hr1G020500 HORVU3Hr1G020520 HORVU3Hr1G051000 HORVU4Hr1G002280 HORVU4Hr1G002290 HORVU4Hr1G015170 HORVU4Hr1G060720 HORVU4Hr1G060760 HORVU4Hr1G063350 HORVU4Hr1G072680 HORVU4Hr1G072700 HORVU4Hr1G072770 HORVU5Hr1G006670 HORVU5Hr1G061160 HORVU5Hr1G061170 HORVU6Hr1G015130 HORVU6Hr1G070230 HORVU6Hr1G077710 HORVU6Hr1G082360 HORVU7Hr1G036470 HORVU7Hr1G036500 HORVU7Hr1G036540 HORVU7Hr1G036570

Legend: - Exon

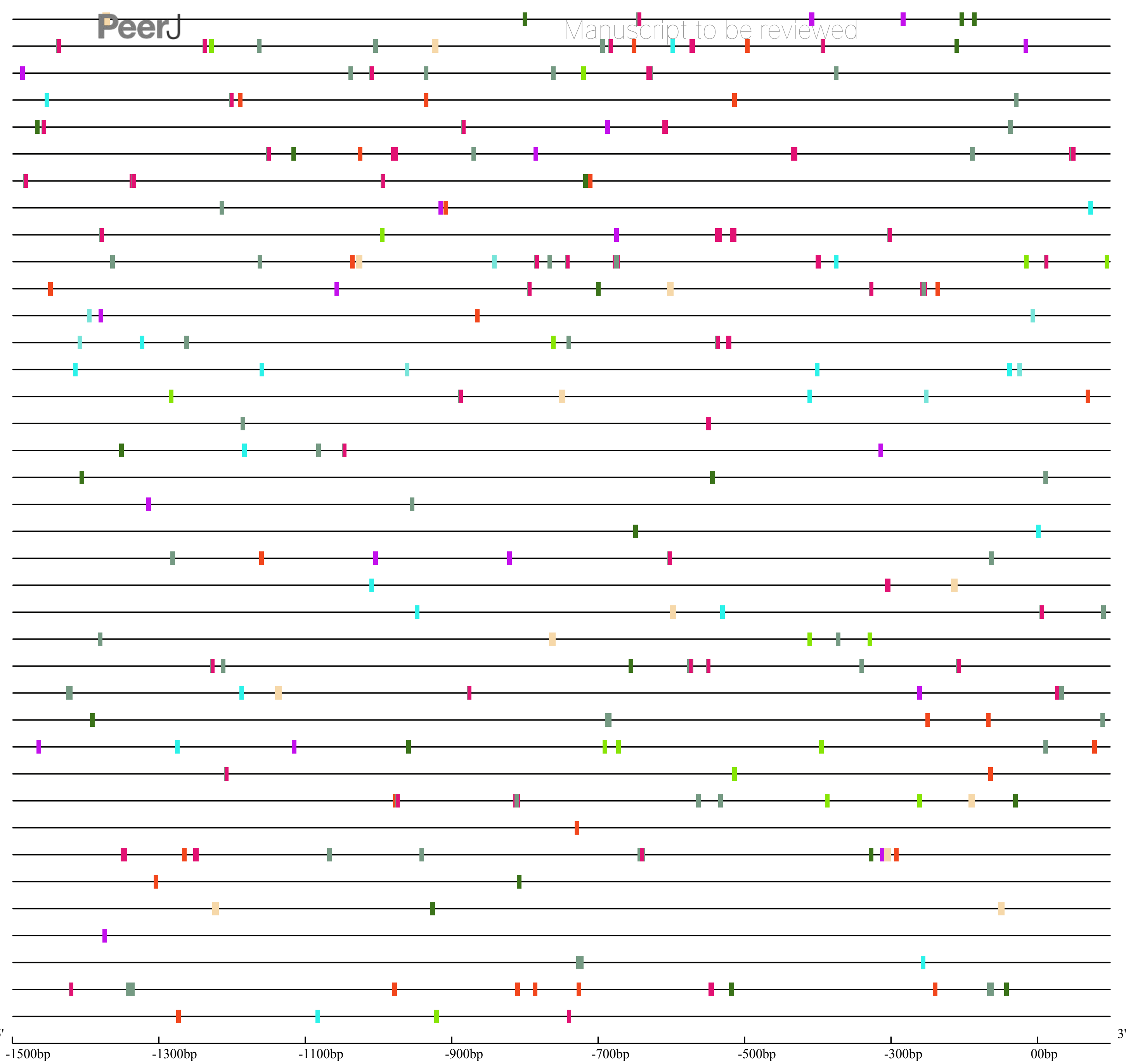

Peer) reviewing PDF | (2018:12:33715:1:2:NEW 12 Mar 2019)
DRE_core G-Box $\quad$ LTR

ABRE CCAAT-box 


\section{Figure 7}

Figure 7 Expression profiles of $\mathrm{HvHsp20}$ genes under various biotic and abiotic stresses.

Green blocks represent decreased and red blocks represent increased transcript levels relative to the respective control. (A) Expression profiles of indentified HvHsp2O genes under arsenic stress in tolerant barley genotype (ZDB160). (B) Expression profiles of HvHsp2O genes in arsenate sensitive barley genotype (ZDB475) under arsenic stress. (C) Expression profiles of HvHsp2O genes in barley shoots under heat stress. (D) Expression profiles of HvHsp2O genes in barley roots under heat stress. (E) Expression profiles of HvHsp2O genes in barley leaves during powdery mildew infection. 
A

Arsenate stress in ZDB160

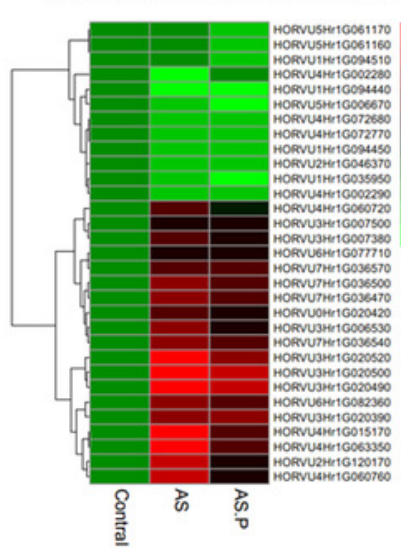

B

Arsenate stress in ZDB475

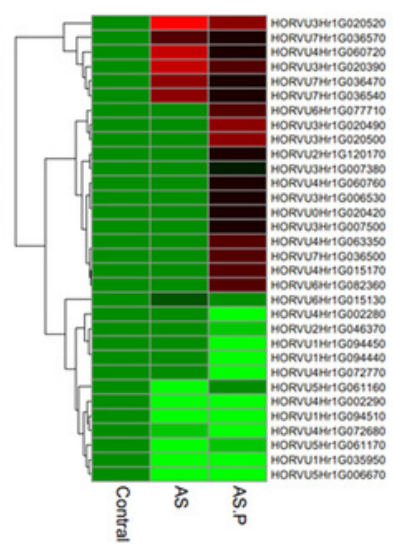

E

Powdery mildew infection
C

Heat stress in shoots

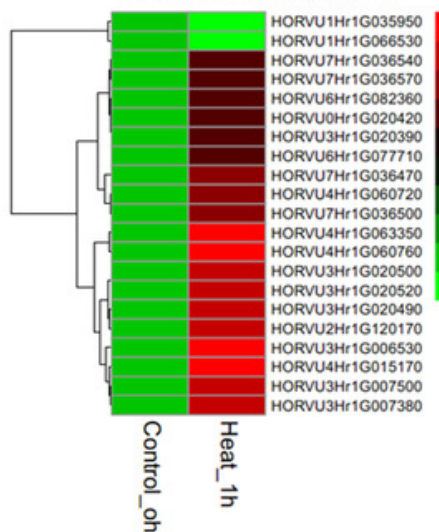

D Heat stress in roots
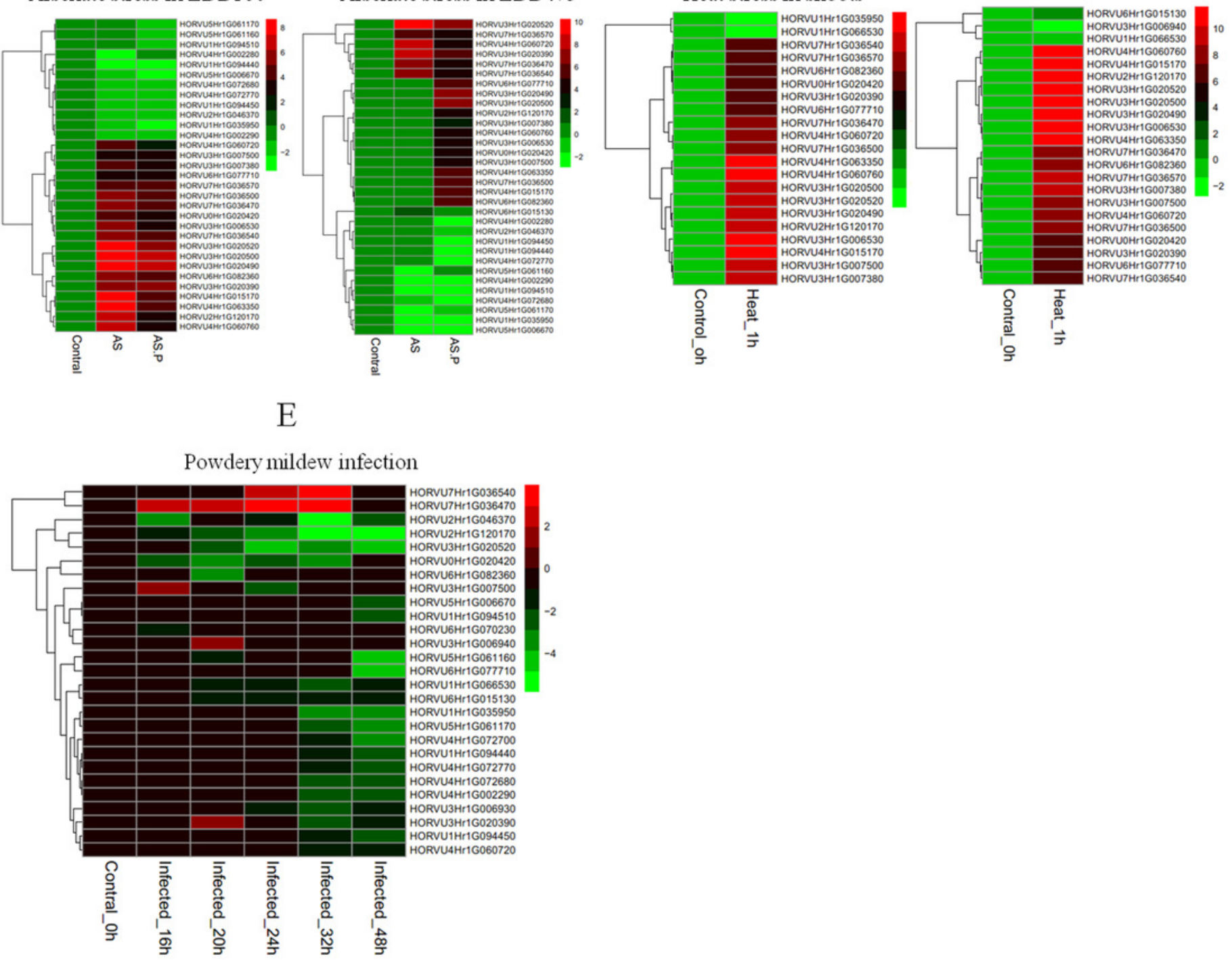